\title{
USING RESPONSE SURFACE METHODOLOGY TO OPTIMISE FACTORS IN CONJOINT EXPERIMENTS
}

\author{
RUBÉN HUERTAS- GARCÍA \\ JUAN CARLOS GÁZQUEZ- ABAD \\ FRANCISCO J. MARTÍNEZ-LÓPEZ \\ IRENE ESTEBAN-MILLAT \\ rhuertas@ub.edu,jcgazque@ual.es,esfjmlopez@ugr.es \\ Universitat de Barcelona, Universidad de Almería, Universidad de Granada, Open University \\ of Catalonia
}

\begin{abstract}
Identifying relevant attributes or variables is the first objective of conjoint analysis in market research. As a result of technological development, today it is common for researchers to use sequential experimental methods for adjusting design factors in successive phases. In particular, in the field of consumer behaviour these models are used predominantly for assessing subjective perceptions relating to the attributes of different products with high sensorial components (e.g. food, drinks and personal care products). This paper illustrates the use of response surface methodology in conjoint experiments, allowing sequential research in which the evaluation of a choice set determines the weight of factors in the next choice set and continues until the optimum combination is achieved. To this end we have carried out a computer simulation to determine the optimal combination of ingredients for a sauce. The simulation shows that the model needs only a few steps to reach the optimal combination of ingredients. This result indicates that response surface methodology can be considered a useful tool in the field of market research and, in particular, in studies on consumer behaviour.
\end{abstract}

\section{Keywords:}

Conjoint Analysis (CA), Response Surface Methodologies (RSM), sequential experiments, sensorial perceptions 


\section{INTRODUCTION}

As a tool for measuring utility, the analysis of consumer preferences remains a central issue in the study of consumer behaviour. Even though both the concept of utility and its measurement have been studied extensively in marketing literature, it remains an ambiguous topic because of the difficulties involved in observing utility and the absence of an absolute measurement scale (Gustafsson et al. 2007).

Over the last few decades the literature has developed and implemented a large number of both compositional and decompositional methods for measuring utility, of which conjoint analysis (CA) is the most important (Green \& Srinivasan 1978). CA has the advantage of combining a simple data collection system with a sophisticated design and evaluation methodology. However, despite the progress in experimental design and modelling literature, a growing number of studies highlight the inherent limitations of this methodology, specifically in estimating the partial worth of the factors or variables it comprises (Gustafsson et al. 2007). While some of the deficiencies are attributable to respondent behaviour (McFadden et al. 2005), the main reason for them is due to breaches of the axioms of the CA model (Louviere et al. 2002). Most empirical CA applications assume two basic propositions: (1) the utility function is an additive polynomial function; (2) the factors or variables are independent of one another (Teichert \& Shehu 2007). When there is failure to comply with the principle of independence between factors, estimating the preference function is much more difficult, given that the possible interactions between attributes need to be considered. This implies much larger designs since they grow exponentially (Street et al. 2005). As a result, many empirical applications used fractional factorial designs without testing the independence of the factors used (Hauser \& Rao 2004).

Within CA, the previous literature has made various proposals in an attempt to overcome the restriction on the assumption of independence between factors. These have been identified through two lines of research: (1) the adaptation of traditional CA models; (2) the proposed special designs based on discrete choice models.

One of the ways of adapting CA is to use sequential experimental methods to improve the efficiency of designs and to alleviate the problem of considering independent factors. Sequential experimental methods involve research at different stages, where information obtained in the early stages of study is used for planning and executing the following stages (Raghavarao et al. 2011). Nowadays, sequential experimental models are particularly timely because - given the advent of market research by internet - conjoint experiments can be developed by computer, programmed sequentially and performed in real time. The literature presents several ways of approaching the sequence in research. One of the first was adaptive conjoint analysis (ACA), as proposed by Johnson (1987), who suggested combining different types of research (e.g. self-assessment questionnaires with an orthogonal design for experiments). ACA is a flexible sequential model that tailors the experimental design to each respondent depending on the previously stated preference ordered in the self-assessment questionnaire (Green et al. 1991). This methodology was refined by Toubia et al. (2003), who developed a polyhedral model to improve the sequence that questions must follow, adapted to each interviewee. This proposal enables information to be gathered as efficiently as possible, taking into account that the primary application of this model is the online survey in which participants are free to leave the questionnaire at any time. More recently, Teichert and Shehu (2007) developed a method that proposes a sequential CA approach using algorithms that change the questions depending on the previous answers, following the principles of Darwin's theory of evolution.

In short, the idea behind sequential experiments is a process of convergence in which the results of the first experiments allow the model to design the profiles that follow. This sequence gives the model the dynamism needed to adjust more readily to consumers' needs and desires. If we are interested in this approach, two extreme options can be considered. Either the information obtained by a simple choice or by an evaluation obtained from a set of choices can be used to set up the new set of choice or, at the 
other extreme, the information obtained from the choice or evaluation of a block can be used to select the next choice set block.

In this paper, we propose to take the first extreme option. In order to do so, we use a model based on response surface methodology (RSM), which enables optimal levels of factors to be achieved. RSM should be considered as an extension of CA, applying it to an evolutionary process. Both models follow the same operating procedure when conducting the experiments. Actually, while CA uses a single experiment to estimate the combination of factors that maximises consumer's utility, RSM, after this first stage, begins an exploration into an interval around the initial results to see if a better adjustment can be found.

The process is as follows. First, we perform an exploratory experiment to determine the significant weight and partial values of the main factors, for example by using an experimental design of resolution III (Louviere 2006). Once these are established, we adjust the values of the levels in a stepwise process to reach the optimum. As each of these steps represents a new experiment, the question arises as to whether the number of steps will be too great in a market research situation to make its application feasible. To provide a possible answer, we present a computer-simulated experiment, where only a few steps are necessary to achieve a combination of factors with a precise degree of approximation to the optimum. The RSM was proposed by Box and Wilson (1951) more than 50 years ago and has been applied to process engineering, medicine, biology and chemistry (Box \& Draper 1987). The main contribution of this paper is to adapt the RSM used in other fields of research to market research. The methodology is based on a sequential process of experiments, where the results of the first serve to improve the fit of the following ones in order to achieve optimal results. These processes with multiple stages were very difficult to adapt to market research using procedures based on personal, telephone or mail surveys. However, the advent of computer-aided conjoint experiments and surveys on the internet makes it possible to apply RSM, as long as the number of steps to reach the optimum is not very great.

The paper is structured as follows. First, we review the conjoint analysis stages that may be most affected by the sequential experimentation. Next, we present the response surface methodology and some previous studies found in customer behaviour literature. Then, using a computer simulation, we present a case study illustrating its use. Finally, we describe the findings and limitations of the experiment.

\section{USING CONJOINT ANALYSIS IN THE FIELD OF CONSUMER BEHAVIOUR}

CA is a decomposition method for estimating the structure of consumer preferences deriving from the overall assessment made on a choice set predetermined by the number of attributes and levels in each attribute (Gustafsson et al. 2007). While in composition methods - such as studies on the expected value - the weight of the parameters and their levels were determined from the direct responses of interviewees, in models of decomposition the results come from the choice or evaluation of different scenarios or profiles proposed by the researcher. Several studies have attempted to evaluate the robustness of both methods, with mixed results (e.g.: Sattler \& Hensel-Bórner, 2007; Gustafsson et al., 2007).

Although the analysis of experiment foundations started in the 1920s, there is a consensus in the literature pointing to the work published by Luce and Tukey (1964) as the beginning of CA, a generic name that includes a set of techniques and methods based on evaluating subjective responses of interviewees and transforming them into parameter estimates. The first work that addressed this emerging methodology in the study of consumer behaviour appeared in the early 1970s (see Green \& Rao 1971). Since this pioneering work an extensive literature has been developed trying to theoretically justify the presence and use of these models. This theoretical development was carried out from two perspectives: (1) the adaptation of the expected value model (Fishbein 1967); (2) the new economic theory of consumer choice (Lancaster 1971). 
Apart from the theoretical difference between these two perspectives, there are also significant methodological differences in the way they carry out the analysis. Thus, while economists have been much more interested in the aggregate implications of a multi-attribute utility structure, psychologists have focused on individual analysis assuming the same functional form for each individual, but allowing variability among the parameters.

Like any market research, marketing experiments require researchers to know a priori the relevant factors (Sawyer et al. 1979). Conjoint analysis also requires pre-exploratory or qualitative research that allows the researcher to select a small number of factors or variables. We can use different ways of identifying the attributes that are relevant for the consumers: focus groups, depth interviews or assessments of the 'product managers', intermediaries and other sources of information, such as the literature itself. In most studies using CA, the basic elements have been decomposed into physical characteristics. And the most frequent use has been by product development teams faced with the decision as to which features to include in the design of a product (Hauser \& Rao 2004). Therefore, when starting with statements made by consumers, they need to be 'translated' into design specifications (Griffin \& Hauser 1993; Huertas-Garcia \& Consolación-Segura 2009). Nevertheless, there is some research that has used the analysis with qualitative attributes to evaluate health services (Hauser \& Urban 1977), and even to construct scenarios in which respondents must offer financial decisions (Shepherd \& Zacharakis 1999).

Once the number of factors has been defined, the process to develop a CA involves six steps (Green \& Srinivasan, 1990):

1. Selection preference model

2. Data collection method

3. Construction of the set of stimuli of the full/partial profile method

4. Presentation of stimuli

5. Measurement scale for the dependent variable

6. Estimation method

Even though the aim of this paper is not to perform a complete review of all aspects of CA, it is important to consider those that are most affected by the use of RSM. The use of this model affects four of the six stages, namely the selection of the preferred model, the method of data collection, the construction of the set of stimuli and the presentation of stimuli.

\section{RESPONSE SURFACE METHODOLOGY (RSM)}

RSM is a collection of statistical techniques used in the development, improvement and optimisation of industrial processes. In particular, it is a very important application in the design, development and formulation of new products (Myers et al. 2009). This methodology has its origins in Box and Wilson (1951); the authors justified its use by the need for efficient experimental procedures to determine the operating conditions of a set of controllable variables that can achieve an optimal response (see also Box \& Draper 1987). Since the 1970s, RSM has gone through several stages of development that have made it adaptable to a wide range of experimental options by eliminating various restrictive assumptions (Myers \& Montgomery, 2002).

Although the RSM has its origin in chemical experimentation and engineering, it has also been used in CA, albeit to a limited extent, for assessing subjective perceptions such as the expected value and sensory analysis. However, it has mainly been applied in the design of physical products, especially those characterised by a strong sensory component (e.g. food and beverages, fragrances, personal care) (Green \& Srinivasan, 1990).

To adapt sequential experimentation to RSM, we must take into account a number of restrictions. To begin with, RSM estimates the slope factor and therefore the model will be a vector type. This would be defined as follows. Let $p=1,2, \ldots, t$ be a set of attributes or factors identified through qualitative 
research. Let $y_{j t}$ be the level of attribute $p$ for stimuli $j$, where $y_{j t}$ can be a continuous variable (e.g. travel time, price) using a ratio scale or interval. Even if the variable is an interval or ratio scale, using a vector model can be a good approximation. Thus, the preference for concept or profile, $s_{j}$, whether measured in response to a rating scale, ranking and/or sensory assessments (Myers \& Montgomery, 2002), is given by

$$
s_{j}=\sum_{p=1}^{P} w_{p} y_{j p}
$$

where $w_{p}$ is the individual weight or average of $p$ 's attribute and its value would indicate the slope.

As for the method of data collection, RSM fits the full profile approach where each concept comprises a combination of levels of each factor variable analysed, and also fits the partial profile. The most used approach is the full profile (Hauser \& Rao 2004). The main argument for this is that it shows the interviewees a more realistic description of stimuli, although at the same time this makes it difficult to answer because they have to consider several factors at once. However, the greatest limitation of this approach is that the amount of information may exceed the capacity of the respondents, forcing them to use heuristics with which they may overvalue certain important attributes at the expense of other less important ones. So the results obtained in a situation like this would not be representative of individuals' behaviour in real life, where they may have more time and motivation to deliberate on the choice between a limited set of alternatives. Therefore, due to the problem of information saturation, the full-profile procedure is usually restricted to five or six factors (Green \& Srinivasan 1978). Likewise, in an environment in which the correlation between factors is important, the full profile is probably the best in terms of predictive validity (Green \& Srinivasan 1990). However, subsequent developments show us that it is possible to achieve efficient results using partial profile designs (Kuhfeld et al. 1994). The best-known model based on the use of partial profiles is Johnson's (1987) ACA. RSM fits both full-profile and partial-profile designs. It also has a wide range of design options that can be adapted into many options put forward by a conjoint analysis Hoke's (1974) design, for instance, proposes a combination of full-profile and partial-profile designs in a single experiment, and Box and Behnken's (1960) designs are based only on combinations of partial profile, and so on..

An important difference between CA and RSM is the scope of the usual analysis in which each of the methods works. Given the importance that CA attaches to variations in preferences between individuals, the model is adjusted for each person instead of analysing aggregate and segmentation data as is usually done in response surface models.

As regards constructing the set of stimuli, RSM is dynamic, which implies an evolution in the levels of each factor. As noted above, sequential experimental methods involve studies in different stages, using information obtained in the previous stages for the planning and execution of the following experiments (Raghavarao et al. 2011). CA is often used to evaluate a hypothetical description of the stimuli of new products. In addition, the number of stimuli depends on the number of factors or attributes considered. Hence, using similar stimuli to those existing in a real situation increases the credibility of the experiment and, therefore, the validity of judgements about preferences. Notwithstanding, Green and Srinivasan (1978) recommend that the range of factors should be somewhat larger than in real life, it should not be so large as to lose credibility among respondents.

As in CA, RSM's full-profile stimuli have the advantage of directly measuring overall judgements about preferences using behaviour-orientated constructs, such as purchase intent, likelihood of choice, and likelihood of changing to a new brand and so on. These are used by CA in a particular way in the context of introducing new products or services (Green \& Srinivasan 1990). This objective is also pursued by RSM (Myers et al. 2009). Fractional factorial designs and other orthogonal plans that exclude or sharply limit the effects of interactions currently dominate the field of market research (Green \& Srinivasan 1990; Hauser \& Rao 2004). RSM is based on sequential experimentation and usually starts by fitting a first-degree polynomial model. However, in the steps of approximation to optimal, RSM allows a second-degree polynomial model, which estimates partial values, interactions and squared factors. 
Another topical issue is the option of using a number of different levels for each factor that makes up a single experiment (Verlegh et al. 2002). In one of the first studies that addressed this issue, Wittink et al. (1982), using both ranking and ratings as dependent variables, showed that the greater the number of levels an attribute has, the greater its relative importance. Thus they found that, by introducing two intermediate price levels on a scale of three levels, the relative price rose by $7 \%$. RSM tends to use the same number of levels for each factor, although it is possible to use a greater number of levels for a factor as long as it is coded for interval $(-1,1)$.

As far as the presentation of stimuli is concerned, the full- and partial-profile approaches have implied variations and combinations of three basic presentations: verbal descriptions, paragraph descriptions and representations in images. RSM fits any type of stimulus. Although the dependent variable originally reflected the physical or chemical changes resulting from experimental application, since the 1970s it has also been used in the sensory assessment of subjective perceptions. One of the first studies using RSM adapted to consumer behaviour was that by Moskowitz et al. (1977), who proposed sequential research to determine the optimal seasoning of different sauce prototypes. This is an example of RSM used in the design of food products. In this experiment, ten types of subjective perception were used as dependent variables, ranging from global ratings at the time of consumption to global assessment a day after of the different assessments: sensory aroma, acidity, sweetness, and so on. Sequential experimentation was also done to find a region of improved response that is the 'steepest ascent to the optimum'.

To the best of our knowledge, there is only one precedent in the market research literature. This is the work by Danaher (1997). He used a full profile with verbal descriptions about a set of attributes for a commercial aircraft flight and a stay in a hotel, and he ended his analysis by determining the exact slope to follow to achieve the optimum. The following two cases show the limitations of RSM in studying consumer behaviour. First, the work carried out by Moskowitz et al. (1977) uses a very small sample of housewives (26 participants between 18 and 49 years) who had to attend three producttasting sessions in a laboratory. Later, Danaher (1997) was able to perform only the exploratory experiment, using a larger sample of 260 and 49 completed questionnaires. However, the possibility of online surveys and computer-aided experiments makes it feasible to adapt CA for quantitative attributes, as it is possible to develop sequential experimentation with the different steps needed to reach the optimum. As pointed out by Raghavarao et al. (2011), and given the dynamic nature of the analysis, virtual environments and computer-aided design are the most suitable for its application, with the advantage that the analysis can be made online or in real time.

In short, the type of experimental design used in RSM is usually a vector function that can be presented in both full and partial profile, in which the factors can be represented by several levels and a range of design options including fractional factorial, hybrids or mixed and with different presentations of stimuli. Therefore the only restrictions for application to conjoint analysis are the use of qualitative variables and the range of encoding levels in the range $(-1,1)$. The model proposed in this paper considers all the factors at two levels, the lowest value encoded as -1 and the highest value as 1 .

In addition, RSM implies a dynamic conception of experimentation that links sequential experimental methods (Raghavarao et al. 2011). Therefore it will require more than a single experiment, so a simple and economical design is very important. It begins by assuming that a first order model (i.e. a flat representation) is a reasonable approximation of the initial region. According to Myers et al. (2009), the methodology of the 'steepest ascent to the maximum' involves the following steps:

1. Fitting a first order model (plane or hyperplane) using an orthogonal design. Two-level designs including the central point are recommended.

2. Calculating the 'path of steepest ascent to the maximum', if maximising the response is wanted. If, on the contrary, the minimal response is wanted, the 'path of steepest descent to the minimum' should be calculated. 
3. Conducting further experiments along the route while we observe the response values. The results will show increasing values of response up to a point where the road bends and increasing values disappear; or the opposite if the goal is minimal response.

4. The point near the maximum (or minimum) located along the route of the 'path of steepest ascent to the maximum' is taken as the basis for a second experiment. The design can again be a first-order design. In this case, tests should be conducted to determine the degree of curvature.

5. The process can continue with a new round of experiments, but if it is seen that the degree of curvature is high, it will be better using a second degree model to fit it.

\section{IMPLEMENTATION OF RSM IN ESTIMATING CA FACTORS: AN EMPIRICAL ANALYSIS USING A SIMULATED EXPERIMENT}

In order to illustrate the proposed procedure, we have used a computer-simulated experiment to generate ideal sensory profiles. The experiment is inspired by the work of Moskowitz et al. (1977), which consisted of an experimenter using a factorial design, enabling a representative range of product variations to be obtained as a result of combining the formulation ingredients. Consumers then test each product and perform a sensory evaluation on a scale. After this, the investigator determines the weight of each factor level, adjusting the results with a first-degree polynomial equation, and then designs the new experiment. This is a common problem in product development involving formulation, which needs to combine two or more ingredients (Myers \& Montgomery, 2002).

In the simulation, we propose three ingredients for the formulation that are manipulated to achieve optimal sensory evaluation. The aim is to evaluate a sauce and there are three factors: acidity, sweetness intensity and degree of saltiness. The degrees of these are changed by the amount of additive added (i.e. vinegar, sugar and salt) to a uniform scale of 0 to 1 for the three ingredients. To start the exploratory experiment, we considered the three variables at around the average, with two levels: as low as 0.4 and as high as 0.6 . This represents a $2^{3}$ factorial experiment, i.e. 8 elementary experiments to which we have added a profile that represents the centre point. Usually, RSM starts with an exploratory experiment in order to reduce the number of factors. It is from this smaller factor of number that we initiate the 'steepest ascent'. The literature recommends considering this first result as the point of origin 'as a confirmation test, to ensure that conditions experienced during the original experiment have not changed' (Myers et al. 2009, p. 185).

The transformation of factors into dummy variables is shown in Table 1 . Since only three variables have been considered, we have chosen a full factorial design of eight experiments with two levels in each factor. This two-level design - also called geometric design (Kuehl 2001) - is one of the most commonly used in exploratory experiments where the number of scenarios is a multiple of 2, i.e. 8, 16, 32, and so on. There are also non-geometric designs, although they are little used because of their complexity (Box, Hunter, \& Hunter, 2005).

Table 1

Factorial design scheme $2^{3}$.

\begin{tabular}{llccc}
\hline & Variable & -1 & 0 & +1 \\
\hline 1 & Acidity & 0.4 & 0.5 & 0.6 \\
2 & Sweetness & 0.4 & 0.5 & 0.6 \\
3 & Saltiness & 0.4 & 0.5 & 0.6 \\
\hline
\end{tabular}

For each of the proposed scenarios, we have simulated 100 consumers, randomly, evaluating each of the nine options as a first-degree additive model of, $\hat{y}=\alpha+\beta-\gamma+u_{i}$, where $\alpha$ is the degree of acidity, $\beta$ the degree of sweetness, $\gamma$ the degree of saltiness and $u_{i}$ random error that is distributed as a 
normal range $(0,5)$. To estimate the accuracy halt at the optimal value predetermined by the researcher, the algorithm calculates the square error (RMSE).

$$
\text { RMSE }=\sqrt{\sum_{k=1}^{n} e_{k}^{2}}
$$

where $e_{k}$ is the difference between the value estimated by the simulator and the optimum predetermined by the researcher, and $k=1,2, \ldots n$, is the number of people simulated. In other words, RMSE includes both types of random variation, one that comes from the computer-generated random numbers and another from the result of the difference between the simulated and the optimal values predetermined by the researcher. Using RMSE as the dependent variable, the objective will determine how many steps the RSM algorithm needs to eliminate the variation explained by the lack of fit and, therefore, for the $u_{i}$ to just pick random error.

Table 2 shows the simulation result obtained by calculating the mean square error.

Table 2

Results of experiment

\begin{tabular}{lcccr}
\hline $\begin{array}{l}\text { Number of } \\
\text { experiment }\end{array}$ & Acidity & Sweetness & Saltiness & Square error (SE) \\
\hline 1 & -1 & -1 & -1 & 782.18 \\
2 & 1 & -1 & -1 & 1141.65 \\
3 & -1 & 1 & -1 & 874.24 \\
4 & 1 & 1 & -1 & 1377.64 \\
5 & -1 & -1 & 1 & 739.14 \\
6 & 1 & -1 & 1 & 1120.19 \\
7 & -1 & 1 & 1 & 840.31 \\
8 & 1 & 1 & 1 & 1375.58 \\
9 & 0 & 0 & 0 & 998.69 \\
\hline
\end{tabular}

To calculate the effect that each quantity of ingredients has on the assessment of the sauce, as a method of adjusting the results we have used the table of contrast coefficients instead of adjusting the model by OLS. This is in order to avoid having to change software during the simulation process. It is a very simple procedure that can be performed with any spreadsheet because the coding system of independent variables converts the moment matrix into an identity matrix, and therefore the fitted regression model is simply $y=X b$. The coded matrix is placed in standard order and multiplied by the vector of results. The standard order is presented in Table 3.

The procedure can be schematically illustrated as follows. We place in the first column of the matrix eight positive ones and finally the central value, zero. Then in the second column, acidity, we place one negative and one positive sign alternating; in the sweetness column we put two positive signs alternating with two negative signs; and finally in the saltiness column the alternating signs are four negative and four positive. In general terms, if the design continues, the $k$-th column will consist of $2^{\mathrm{k}-1}$ negative signs followed by $2^{\mathrm{k}-1}$ positive signs, ending with the core values, zeros.

Once these have been ordered, we have calculated the matrix of contrast coefficients; each row of values has been multiplied by the square error in that row. Table 4 illustrates the procedure. To estimate the effects we can only add the values of each column and divide by the appropriate divisor: 8 for the first row and 4 for the remaining rows. The first column provides the overall average of all the observations and the other column factors are identified by acidity, sweetness and saltiness.

Table 3 
Table in standard order

\begin{tabular}{lccccr}
\hline $\begin{array}{l}\text { Number of } \\
\text { experiment }\end{array}$ & Average & Acidity & Sweetness & Saltiness & Square error (SE) \\
\hline 1 & 1 & -1 & -1 & -1 & 782.18 \\
2 & 1 & 1 & -1 & -1 & 1224.36 \\
3 & 1 & -1 & 1 & -1 & 874.24 \\
4 & 1 & 1 & 1 & -1 & 1.562 .97 \\
5 & 1 & -1 & -1 & 1 & 718.78 \\
6 & 1 & 1 & -1 & 1 & 1216.42 \\
7 & 1 & -1 & 1 & 1 & 912.49 \\
8 & 1 & 1 & 1 & 1 & 1560.63 \\
9 & 0 & 0 & 0 & 0 & 998.69 \\
\hline
\end{tabular}

Table 4

Calculus of part worth by contrast coefficients

\begin{tabular}{crrrr}
\cline { 1 - 4 } & Average & \multicolumn{1}{c}{ Acidity } & \multicolumn{1}{c}{ Sweetness } & \multicolumn{1}{c}{ Saltiness } \\
\hline & 782.1825738 & -782.1825738 & -782.1825738 & -782.1825738 \\
& 1224.360214 & 1224.360214 & -1224.360214 & -1224.360214 \\
& 874.2385556 & -874.2385556 & 874.2385556 & -874.2385556 \\
& 1562.967695 & 1562.967695 & 1562.967695 & -1562.967695 \\
& 718.7792558 & -718.7792558 & -718.7792558 & 718.7792558 \\
& 1216.418861 & 1216.418861 & -1216.418861 & 1216.418861 \\
& 912.4940999 & -912.4940999 & 912.4940999 & 912.4940999 \\
& 1560.63063 & 1560.63063 & 1560.63063 & 1560.63063 \\
& 0 & 0 & 0 & 0 \\
\hline Sum & 8852.071885 & 2276.682915 & 968.5900759 & -35.42619046 \\
\hline Divisor & 8 & 4 & 4 & 4 \\
\hline Average (in & 11.06508986 & 5.691707288 & 2.42147519 & -0.088565476 \\
units xi & & & & \\
/100) & & & & \\
\hline
\end{tabular}

The first-order model derived from the experimental design is:

$$
\hat{y}=11,065+5,691 \alpha+2,421 \beta-0,088 \gamma
$$

where $\hat{y}$ is RMSE, $\alpha$ is the degree of acidity, $\beta$ the degree of sweetness and $\gamma$ the degree of saltiness in generation of square error.

Once the first-order model has been determined, we need to set the path to achieve the values to minimise the RMSE. To calculate the 'path of steepest descent to the minimum', we follow the algorithm below (Myers \& Montgomery, 2002):

1. We choose the step size or range that we move forward down the slope, called $\Delta x_{i}$. To do this we selected the variable with the regression coefficient of greatest absolute value $\left|b_{i}\right|$.

2. The step size in the other variables will be:

$$
\Delta x_{j}=\frac{b_{j}}{b_{i} / \Delta x_{i}}, j=1,2, \ldots, k, i \neq j
$$

Finally, we need to convert the coded variables into natural variables. For the example considered, we select $b_{1}=\alpha$ as a variable to define the size of the step, taking one unit increment, $\Delta x_{1}=1$, which 
corresponds to 0,1 of variation in the amount of acidity in variable $\alpha$. From equation (2) we derive the sizes of the other steps:

$$
\begin{gathered}
\Delta x_{2}=\frac{2,421}{5,691}=0,425 \\
\Delta x_{3}=\frac{-0,088}{5,691}=-0,015
\end{gathered}
$$

Table 5 shows the steps down the slope for both the coded and the natural variables. The strategy implies that we perform experiments all the way down to the step when the mean square error, instead of going down, grows back. This has occurred when the base value is subtracted three steps or increments.

Table 5

The steeps dawn the slope

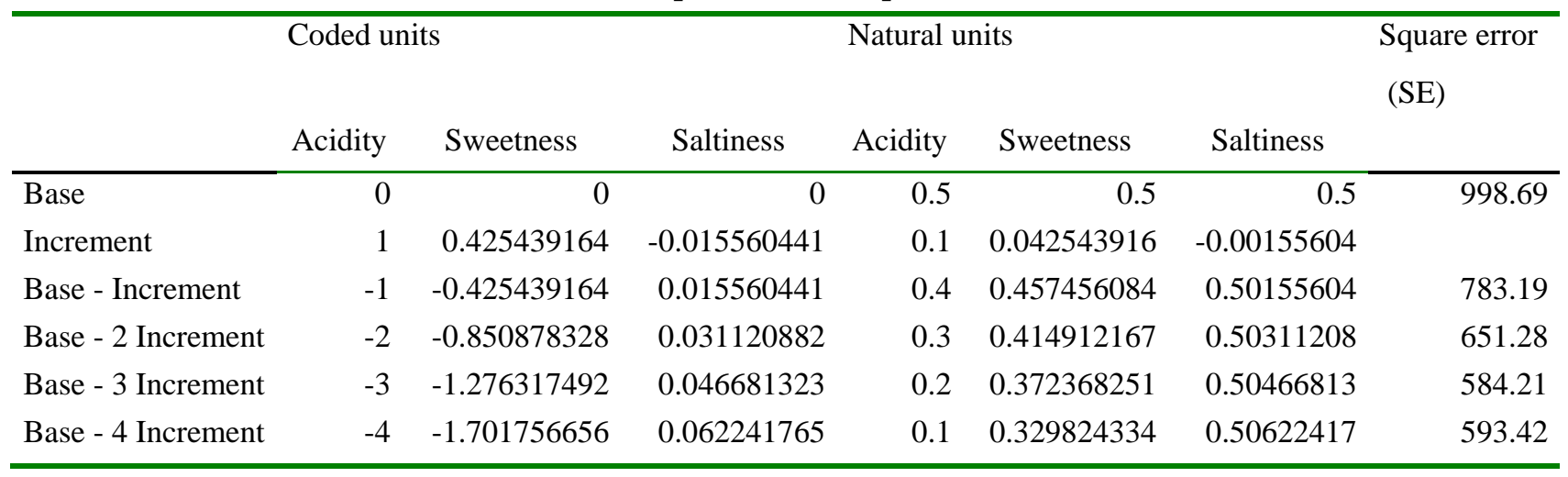

In most cases, the result obtained provides a good approximation to the optimum. However, if improving accuracy is wanted, a second experiment can be performed in a range around this point. In our case, this second time a scan interval of 0.05 points has been taken. The transformation of these variables into coded units is shown in Table 6, and the experimental design followed is reported in Table 7. From here, for each of these new scenarios we have re-estimated by computer simulation the assessment of 100 consumers who carry out a sauce sensory analysis, and have also calculated the mean square error. The design can again be a first-order design and reuse the same process of calculating the 'path of steepest descent to the minimum'

Table 6

Scheme of new factorial design $2^{3}$.

\begin{tabular}{llrrr}
\hline & Variable & \multicolumn{1}{c}{-1} & \multicolumn{1}{c}{0} & \multicolumn{1}{c}{+1} \\
\hline 1 & Acidity & 0.15 & 0.2 & 0.25 \\
2 & Sweetness & 0.160 & 0.372 & 0.585 \\
3 & Saltiness & 0.497 & 0.505 & 0.512 \\
\hline
\end{tabular}

Table 7

\begin{tabular}{ccc}
\multicolumn{3}{c}{ Design } \\
\hline 1 & 2 & 3 \\
\hline-1 & -1 & -1 \\
+1 & -1 & -1 \\
-1 & +1 & -1 \\
+1 & +1 & -1 \\
-1 & -1 & +1 \\
+1 & -1 & +1 \\
-1 & +1 & +1 \\
\hline
\end{tabular}




\begin{tabular}{rrr}
\hline+1 & +1 & +1 \\
0 & 0 & 0 \\
\hline
\end{tabular}

After two rounds estimating the 'path of steepest descent to the minimum', the values obtained for $\alpha=$ acidity, $\beta=$ sweetness and $\gamma=$ saltiness are shown in Table 8 and compared with the optimal values predetermined by the researcher.

Table 8

Estimation of part worth of $\alpha, \beta$ y $\gamma$

\begin{tabular}{lccc}
\hline Origin of values & $\alpha$ & $\beta$ & $\gamma$ \\
\hline Estimated values & 0.15 & 0.372 & 0.512 \\
& & & 0.51 \\
\hline
\end{tabular}

In short, we have needed six steps to achieve a close approximation to the optimal set using a computer simulation system. This result is a reasonable number of steps for adapting RSM to sequential experimentation. Moskowitz et al. (1977), for example, needed three sessions to gather enough information to define optimal levels of ingredients, and Danaher (1997) only made the first step in determining the slope factor. Therefore, this work opens up the possibility of using RSM in market research in such a way that, with just a few stages, higher levels of precision can be reached in the answers than in previous models.

\section{CONCLUSIONS}

The aim of this study was to illustrate the process of using response surface models, and to show how these can be adapted to the sequential experimentation processes commonly used in market research analyses. RSM has two stages: one exploratory, in which the model is built, and the second one, in which the first model is refined. The refinement starts by exploring an interval around the results to discover the slope and then explores the steepest ascents to reach a local optimum. In addition, the model can change the search direction, for instance to explore the degree of curvature, to adjust the model to a second-degree equation in order to estimate curvatures.

The response surface model proposed in this study offers two important advantages. First, it provides a statistical technique that has been validated by the literature on engineering, chemistry and medicine, and that can be adapted to models of sequential experimentation. Second, it provides the possibility not only of calculating the partial values of the factors but also of finding their optimal combination. Furthermore, it is a methodology that fits computer-assisted research, which enables the researcher to adapt it according to the research results obtained, allowing the research process to reach the optimum.

To determine the feasibility of RSM applied to market research, it is important to establish the number of steps to be performed by the researcher in a sequential experiment to achieve the optimum. With illustrative purposes, we have performed a computer simulation on the composition of a sauce; six steps have been needed to achieve a fairly accurate approximation to the optimum, using a linear model without interactions. The experiment was repeated eight times, and each time

in eight steps. The option that we have presented in this paper was one of the most pessimistic, in the sense that it required more steps than the others. It was chosen because it provides the best illustration of the procedure. However after the six steps, the increases in accuracy were negligible

However, the RSM model would have allowed a more precise fit using a second-degree polynomial equation, and achieving a degree of precision normally found in industrial and chemical experiments. Notwithstanding, when we work with sensory analysis based on the subjective perceptions of consumers, reaching such a high degree of precision does not make much sense; i.e. values very close to optimal with only six steps is not an excessive burden on respondents in a computer-aided 
experiment. For instance, in ACA studies, Sawtooth Software proposes that respondents complete questionnaires in four phases, each containing multi-stage questions. Also, in another study where they applied discrete choice models, it was shown that respondents can request up to 20 choice tasks without degrading the quality of the data collected; although with more than 20 choices some reliability loss was observed because respondents tended to complete the questionnaires much faster. In addition, though it is an undisputed value of the sample size, we can gather more information by increasing the number of tasks per respondent. Within the range of between 10 and 20 jobs, doubling the number of tasks per respondent is more effective than increasing precision with twice the number of respondents (Johnson, \& Orme, 1996).

In this paper, we have proposed some simple assumptions, since this is a mixture experiment. In particular, as indicated by Raghavarao et al. (2011, p. 32): 'An experiment in which the factors are the ingredients or constituents of a mixture is called a mixture experiment ... [and] the response is a function of the proportions of each ingredient, with the amount of each ingredient usually measured by weight, volume, or the like.' So far the implementation of response surface models, more similar to CA, has been carried out to evaluate the sensory perceptions of food products. This proposal allows scientists and food technologists to determine the proper formulation of new products they want to launch. RSMs have been used to provide information from consumers to calibrate the composition of ingredients, to achieve an optimal formulation in a relatively short period of time, and with cost savings when compared with other traditional methods of trial and error (Moskowitz et al. 1977). Therefore, for any product that requires a mixture of components, for example in designing a web, RSM can determine the optimal proportions of design elements used to construct the homepage (i.e. banner, general web body, and so on) for different market segments.

In our study we used a sauce in tribute to the early studies that were the first to use RSM to collect consumers' assessments. However, the model can be used in any type of experiment where the dependent variable can be a mean, proportion, etc., allowing for discrete choice models. But, in order to apply this methodology, the independent variables should be quantitative to enable the researcher to adjust their size depending on the evolution of the stated preferences. This does not mean that one cannot also use mixed designs - ones that combine qualitative and quantitative variables in one experiment - but it should be borne in mind that the steepest ascent process runs only with the quantitative variables.

\section{LIMITATIONS AND FUTURE RESEARCH}

This work is in its early stages and has a number of limitations. First, the design we have used in this simulation is very simple - we have used only three factors at two levels plus a central point. This study departs from the CAs commonly used with six to seven factors (Wittink \& Cattin 1989). Second, the data considered in the simulation were a completely homogeneous distribution derived from random number generation by computer. Therefore, a possible extension would be the use of more complex models with a greater number of factors and levels carried through a field, and considering the possibility of extracting market segments.

Since RSM allows different forms of application, from an individual analysis to an aggregate analysis, it offers different ways of approaching the segmentation concept. One is to try to identify different groups of consumers who follow different paths to achieve optimal combinations of ingredients. It is clear that in markets not everyone has the same liking for the sauce. Within the response surface methodology there are a variety of designs, such as Hoke's (1974) mixed-profile designs or Box and Behnken's partial-profile designs, which allow individual estimates. In this case, a two-stage segmentation procedure would be applied. However, in the literature, there is a considerable amount of criticism of the use of individual analyses, especially when researchers use them to form segments. For instance, Elrod et al. (1992) pointed out the instability and bias in the individual-level part-worth estimates, and Kamakura et al. (1994) reported that, in two-stage segmentation procedures, researchers often ignore the estimation error associated with the part-worth estimates from the first stage. All these factors generate a poor adjustment in the models at individual level. 
The other approach, which is focused on an aggregate level of analysis, allows some of the restrictions from the individual-level analysis to be overcome. On the one hand, it allows the use of a larger number of factors without the respondents' burden problem, because the full factorial analysis is divided into smaller blocks and each respondent assesses only one block arrangement; at the end of the experiment, the researcher makes an aggregate analysis among all respondents (this procedure is similar to Adaptive Conjoint Analysis). On the other hand, using the aggregate level allows the compilation of a number of answers from each profile, thus enabling the researcher to make a better fit of the model. One disadvantage is that it adds a heterogeneous group of people to the aggregation process. As a result, this type of analysis requires the use of groups considered, a priori, to be homogeneous based on their observable characteristics (e.g. their socio-demographics) and then estimates consumer preferences aggregate by segment.

The success of this approach depends on the strength of the relationship between socio-demographic characteristics and consumer preferences. If the relationship is weak, the approach generates spurious estimations of factors, as reported by DeSarbo et al. (1992) and Elrod et al. (1992). To try to alleviate the limitations caused by the assumption of preference homogeneity across consumers, a series of latent class segmentation analyses have been proposed (e.g. Kamakura et al. 1994; Ramaswamy \& Cohen 2007). All these suggestions are beyond the scope of this investigation.

Future experiments are planned with computer-aided sequential enabling, which would allow us to see empirically the internal and external validity of the proposed method. Thus, though experiments with living creatures can generate much higher variance than those in other fields of science (see Gilmour 2006), we think that, for this kind of marketing problem, these computer-based experiments can provide similar results to those generated by experiments with people

\section{Bibliografía}

BOX, G. E. P. \& DRAPER, N. R. (1987). Empirical Model Building and Response Surfaces. New York: Wiley.

BOX, G. E. P., HUNTER, J. S., \& HUNTER, W. G. (2005). Statistics for experimenters :Design, innovation, and discovery (2nd ed.). Hoboken: Wiley.

DANAHER, P. J. (1997). Using conjoint analysis to determine the relative importance of service attributes measured in customer satisfaction surveys. Journal of Retailing, 73(2), 235-260. doi:DOI: 10.1016/S0022-4359(97)90005-1

FISHBEIN, M. (1967). Readings in attitude theory and measurement. New York: Wiley.

GREEN, P. E., \& RAO, V. R. (1971). Conjoint measurement for quantifying judgmental data. Journal of Marketing Research, VIII, 355-363.

GREEN, P. E., \& SRINIVASAN, V. (1978). Conjoint analysis in consumer research: Issues and outlook. Journal of Consumer Research, 5, 103-123.

GREEN, P. E., \& SRINIVASAN, V. (1990). Conjoint analysis in marketing research: New developments and directions. Journal of Marketing, 54(4), 3-19.

GREEN, P. E., KRIEGER, A. \& AGARWAL, M. (1991). Adaptive Conjoint Analysis, some Caveats and Suggestions, Journal of Marketing Research, 23, 215-222.

GUSTAFSSON, A., HERRMANN, A., \& HUBER, F. (2007). Conjoint measurement :Methods and applications (4th ed.). Berlin etc.: Springer.

HAUSER, J., \& RAO, V. (2004): Conjoint Analysis, Related Modeling and Applications, in: Wind, Y., \& Green, P. (Eds.), Marketing Research and Modeling, Progress and Prospects, A Tribute to Paul Green, Dordrecht. 
JOHNSON, R. M. \& ORME, B. K. (1996): How Many Questions Should You Ask in Choice-Based Conjoint Studies? Sawtooth Software. Research Paper Series, Sawtooth Software, Inc. 1996

KUEHL, R. O. (2001). Diseño de experimentos :Principios estadísticos de diseño y análisis de investigación. Australia etc.; Madrid: Thomson Learning.

LANCASTER, K. (1971). Consumer demand: A new approach. New York: Columbia University Press.

LOUVIERE, J., STREET, D., CARSON, R., AINSLIE, A., DESHAZO, J., CAMERON, T., HENSHER, D., KOHN, R., \& MARLEY, T. (2002): Dissecting the Random component of Utility, Marketing Letters, 13 (3), 177-193.

LOUVIERE, J. (2006): What you don't know might hurt you: Some unresolved issues in the Design and Analysis of Discrete Choice Experiments, Environmental \& Resource Economics, 34, 173-188.

LUCE, R. D., \& TUKEY, J. W. (1964). Simultaneous conjoint measurement: A new type of fundamental measurement. Journal of Mathematical Psychology, 1, 1-27.

MC FADDEN, D.L., BEMMAOR, A. C., CARO, F. G., DOMINITZ, J., JUN, B-H., LEWBEL, A., MATZKIN, R. L., MOLINARI, F., SCHWARZ, N., WILLIS, R. J., \& WINTER, J.K. (2005): Statistical Analysis of Choice Experiments and Surveys. Marketing Letters, 16 (3/4), 183-196

MOSKOWITZ, H. R., STANLEY, D. W., \& CHANDLER, J. W. (1977). The eclipse method: Optimizing product formulation through a consumer generated ideal sensory profile. Canadian Institute of Food Science Technology Journal, 10(3), 161-168.

MYERS, R. H., \& MONTGOMERY, D. C. (2002). Response surface methodology :Process and product optimization using designed experiments (2nd ed.). New York: John Wiley \& Sons.

RAGHAVARAO, D., WILEY, J. B., \& CHITTURI, P. (2011). Choice-based conjoint analysis: Models and designs (1 edition ed.) Chapman and Hall/CRC.

SAWYER, A. G., WORTHING, P. M. AND SENDAK, P. E. (1979): “The Role of Laboratory Experiments to test Marketing Strategies” Journal of Marketing, Vol. 43 (3); summer; pp. 60-67.

STREET, D., BURGUESS, L., \& LOUVIERE, J. (2005): Quick and Easy Choice Sets, Constructing Optimal and Nearly Optimal Stated Choice Experiments, International Journal of Research in Marketing, 22 (4) 459-470.

VERLEGH, P. W. J., SCHIFFERSTEIN, H. N. J. \& WITTINK, D. R. (2002): Range and number-of-levels effects in derived and stated measures of attribute importance, Marketing Letters, 13 (1), 41-52.

WITTINK, D. R., \& CATTIN, P. (1989). Commercial use of conjoint analysis: An update. Journal of Marketing, 53, 91-96.

WITTINK, D. R., KRISHNAMURTHI, L., \& NUTTER, J. B. (1982). Comparing derived importance weights across attributes. The Journal of Consumer Research, 8(4), pp. 471-474. Retrieved from http://www.jstor.org/stable/2489037 